\title{
Microwave Noninvasive Blood Glucose Monitoring Sensor: Human Clinical Trial Results
}

\author{
Heungjae Choi ${ }^{1}$, Steve Luzio $^{2}$, Jan Beutler ${ }^{3}$, and Adrian Porch ${ }^{1}$ \\ ${ }^{1}$ Cardiff University, CF24 3AA Wales, United Kingdom \\ ${ }^{2}$ Swansea University Medical School, SA2 8PP Wales, United Kingdom \\ ${ }^{3}$ University of Luxembourg, L-4365 Luxembourg
}

\begin{abstract}
In this paper, a microwave non-invasive blood glucose monitoring system operating at around $1.4 \mathrm{GHz}$ is designed and its performance in terms of accuracy and repeatability is evaluated by a clinical trial involving 24 human subjects, with and without diabetes. Direct comparison with the most accurate benchtop glucose analyzer shows the exceptional accuracy and repeatability of the proposed system.

Index Terms-Clinical trial, blood glucose, microwave, non-invasive, wearable sensors.
\end{abstract}

\section{INTRODUCTION}

Diabetes represents one of the largest health concerns of the $21^{\text {st }}$ century. It affects $7 \%$ of the world's population and is on the rapid rise, being estimated to affect 1 in 10 adults by 2040 [1]. The risk of diabetes-related complications can be significantly reduced by maintaining tight control over blood glucose. At present many patients make regular measurements of their blood glucose. This usually involves withdrawing a blood sample via an invasive finger-prick procedure. This is both painful and inconvenient, resulting in reduced patient compliance and less effective glycemic control, or by other invasive methods, such as continuous glucose monitoring systems (CGMs), with associated risk of infection. As a result, there is an ever-growing demand for a reliable non-invasive glucose monitoring system.

Various types of minimally or non-invasive blood glucose monitor (NIBGM) devices have been investigated across the full range of the electromagnetic spectrum, including radio waves, microwaves ( $\mathrm{GHz}$ to $\mathrm{THz}$ range), infrared and visible radiation. In the radio and microwave range, near-field monitoring techniques have been widely used in the non-invasive characterization of material dielectric properties. Some examples include coplanar waveguide sensor integrated with microfluidic system [2], a spiral-shaped microstrip transmission line sensor [3], microstrip patch/slot antenna-based sensor [4][5], and millimeter-wave waveguide transmission measurement system [6]. There are many reported results showing that the complex dielectric constant changes with the presence and fluctuation of blood glucose concentration in the water/glucose system and in humans [7][8]. In [9], we have shown that the prototype microwave NIBGM sensor is effective in monitoring the fluctuation of blood glucose level in a laboratory environment.

In this paper, a new non-invasive blood glucose monitoring system (NIBGM) is designed, and to evaluate the performance

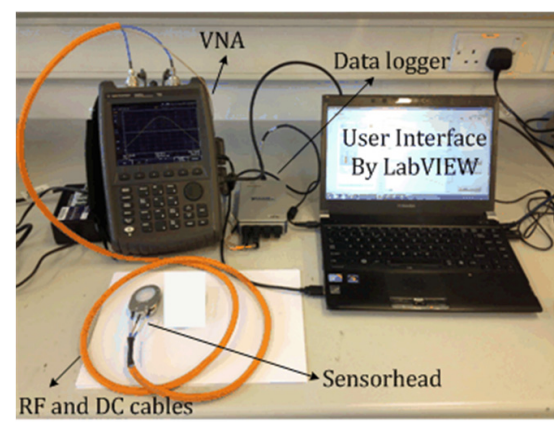

(a)

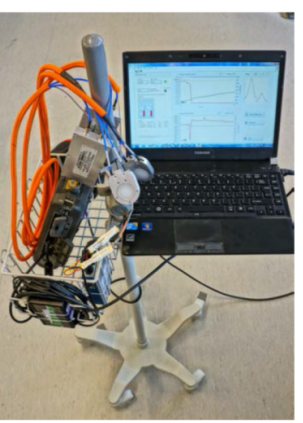

(b)
Fig. 1. Photographs of the (a) proposed microwave non-invasive blood glucose monitoring system, and (b) setup on a medical trolley for clinical trial.

of the proposed NIBGM system, a human clinical trial involving 24 non-diabetic and diabetic subjects has been designed and carried out, and the results are presented.

\section{System DESIGN AND DATA ANALYSIS}

\section{A. System Design}

The basic experimental system consists of four elements as shown in Fig. 1 (a), a sensor head, a portable vector network analyzer (VNA, N9923A, Keysight Technologies), a data logger (NI 9219 CompactDAQ, National Instruments), and a PC with control software (LabVIEW, National Instruments). The VNA applies a swept frequency signal with an output power of about $1 \mathrm{~mW}$ to the sensor head and also measures the transmitted power. The changes in resonant frequency, $3 \mathrm{~dB}$ bandwidth, and insertion loss are tracked in real-time by fitting to a skewed Lorentzian function. The system is set to produce data points every 2 seconds.

The proposed NIBGM system measures the dielectric properties of the top few mm of epidermis, dermis and subcutaneous tissue in the abdominal area. As shown in Fig. 2 (a) in more detail, the sensor head is a microwave resonant circuit based on a split-ring resonator. The radius of the ring is $12 \mathrm{~mm}$, and has a gap of $1 \mathrm{~mm}$ on one side, giving a resonant frequency of around $1.4 \mathrm{GHz}$. The ring is supported by perfluoroalkoxy alkane discs (PFA, Goodfellow) which have very low microwave loss, where the thickness of the discs are used to adjust the distance of the ring from human skin to optimize sensitivity and 


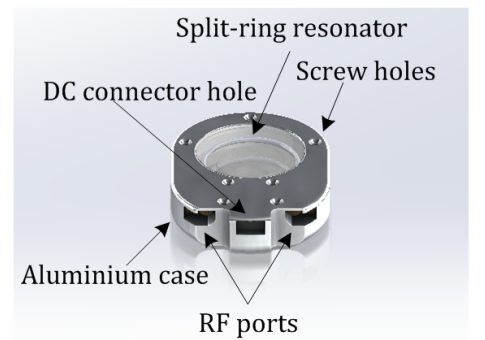

(a)

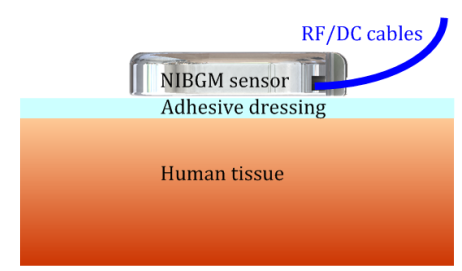

(b)

Fig. 2. (a) 3D drawing of the wearable microwave sensor head. Overall dimensions are $44 \times 60 \times 12 \mathrm{~mm}^{3}$, and (b) illustration showing how the sensor is attached to human skin with open face facing towards skin for sensing.

the dielectric loading effect. The specific parameters of the resonant circuit (its resonant frequency and bandwidth) are affected by the dielectric properties of the tissues within its vicinity. The ring resonator is enclosed in an aluminum case with one face open, where it is attached to the human skin via an adhesive dressing (1504XL, 3M) as shown in Fig. 2 (b). Therefore there is no interference from external and ambient wireless signals, and also no microwave signal is radiated from the aluminum case. A temperature monitoring element, a thermistor, is also placed in the sensor head to monitor the fluctuation of skin temperature. The overall dimensions of the sensor head are $44 \times 60 \times 12 \mathrm{~mm}^{3}$. It is connected to the VNA by lightweight and flexible microwave cables (Multiflex, Huber and Suhner). Two identical sensor units have been fabricated (labelled units 1 and 2) for the clinical trial. Sensor performance evaluation including resonant characteristics, equivalent circuit models and finite element simulation model, and some interference test results can be found in [9].

\section{B. Data Analysis}

Microwave data obtained from the VNA and the temperature data obtained from the data logger are synchronized and combined with a time stamp derived from the system time to produce an output data file. Raw microwave data are temperature calibrated in this step. Around 450 microwave data points collected over 15 minutes are averaged and this is synchronized to the time stamp on the blood glucose data. Reference blood glucose values are measured at intervals of 15 minutes, as described in the next section. Averaged microwave data readings are extracted at the same timings as for the blood glucose measurements. Microwave data are converted to the estimated blood glucose concentration by using subject-specific linear correlation coefficient calculated by the glucose concentration before and after glucose uptake. These two sets of values are plotted on the same graph using the same time base (time domain graph) and versus each other (correlation chart).

The strength and direction of the correlation between microwave readings (candidate method) and the reference blood glucose values (comparative method) are quantified by using Pearson's linear correlation coefficient $R$, and the accuracy is validated by mean absolute relative difference (MARD), which is defined as

$$
\operatorname{MARD}=\frac{1}{N} \cdot \sum_{i=1}^{N} \frac{\left|\hat{G}_{i}-G_{i}\right|}{G_{i}},
$$

where $\widehat{G}_{l}$ is a blood glucose concentration estimated by the candidate method, $G_{i}$ is a reference blood glucose concentration measured by the comparative method, and $N$ is the number of samples. MARD is one of the most widely used figures when evaluating and comparing accuracy. Both Clarke Error Grid and Parkes Error Grid are also widely accepted as means of visualizing the accuracy of a device, where each zone on the grids has a certain clinical importance [10].

\section{ClinicAL TRIAL AND RESUltS}

\section{A. Study Protocol}

An approval for a clinical trial was obtained from the UK Medicines and Healthcare products Regulatory Agency (MHRA) before commencing subject recruitment. People with the following conditions were excluded from the recruitment of the test subjects: evidence of clinically relevant disease, known hematological diseases or hemoglobin $<12 \mathrm{~g} / \mathrm{dL}$, pacemaker, pregnancy. A single center study involving 24 subjects with and without diabetes was designed and carried out with 8 non-diabetic and 16 diabetic subjects (4 of type 1 and 12 of type 2).

On each day of the study, after an overnight fast, each subject attended for a period of approximately 5 hours during which blood samples were taken for measurement using a conventional laboratory glucose analyzer (YSI 2300 Glucose and Lactate Analyzer), to compare with the NIBGM. Subjects consumed a $75 \mathrm{~g}$ oral glucose drink in order that fasting and postprandial blood glucose samples could be measured over a wide range of blood glucose concentrations (typically 70 to 450 $\mathrm{mg} / \mathrm{dL}$ ). No adverse effects were reported. During the pre-sampling period on each day of the study, each subject was fitted with an indwelling cannula, sited into an antecubital fossa vein in the subject's forearm in order to take samples throughout the study period. The cannula was attached via a three way tap to slow running saline $(0.154 \mathrm{nmol} / \mathrm{L})$ infusion to maintain the patency of the vein. The NIBGM sensor was then fixed securely to an abdomen site below the ribcage, using the $3 \mathrm{M}$ adhesive. Each sensor was warmed to $33^{\circ} \mathrm{C}$ prior to body fixing to ensure that the time for them to reach thermal equilibrium with the subject's body was minimized. Blood samples (1 $\mathrm{mL}$ each) were taken every 15 minutes with a time stamp over the course of 3 hours. Subjects continued to take their usual medication 
throughout the study period. Subjects were allowed to walk around, although the range was quite limited due to the medical trolley, as shown in Fig. 1 (b).

\section{B. Results}

Fig. 3 shows oral glucose tolerance test results of one of the test subjects, plotted as a function of time and compared with the reference blood glucose concentration. It is clear to see that the estimated glucose concentration by the proposed NIBGM system matches well the rate of increase of glucose concentration after the glucose intake at $t=60 \mathrm{~min}$. It is worth noting that there is no time delay between the two measurements, which proves that the proposed microwave NIBGM sensor penetrates deep enough into the skin and directly interacts with blood. To evaluate the correlation between the two measurements, the same data are plotted as a scatter chart and shown in Fig. 4. The linear correlation coefficient is 0.98 and the MARD of this specific data set is only $3.2 \%$, which shows exceptional accuracy of the proposed measurement. To prove the repeatability, all the data from 24 subjects are plotted in a Clarke Error Grid and shown in Fig. 5. Most of the data are scattered in zones A and $\mathrm{B}$, and only 4 points out of 214 points exist in zone $\mathrm{C}$, with no data lying in zones $\mathrm{D}$ and $\mathrm{E}$ at all. The data points in zone $\mathrm{C}$ and near the zone B-C boundary are due to unexpected movement of some test subjects (when going to toilet, etc.). Table I summarizes the MARD performance for all 24 subjects (12.5\%), 12 subjects measured by sensor unit $1(11.0 \%)$, and the remaining 12 subjects measured by sensor unit $2(14.0 \%)$. This shows that there are device-to-device variations, possibly due to the handbuilt components and physiological difference between the test subjects.

TABLE I

Mean AbSolute Relative Difference Analysis

\begin{tabular}{ccc}
\hline All 24 subjects & 12 subjects by unit 1 & 12 subjects by unit 2 \\
\hline \hline $12.5 \%$ & $11.0 \%$ & $14.0 \%$ \\
\hline
\end{tabular}

\section{CONCLUSION}

The efficacy of the proposed microwave NIBGM system was evaluated by a human clinical trial involving 24 healthy and diabetic subjects. The error grid and MARD results look very promising but the current system is quite restrictive in its use in that it involves the use of portable network analyzer and is not fully wearable/portable. Our future work is to integrate the proposed system into a fully wearable sensor.

\section{ACKNOWLEDGMENT}

This work was supported by the Wellcome Trust [WT096848MA] and Cardiff University through its Cardiff Partnership Fund. Dr. Maggi Tebrake and 3M Health Care are thanked for their provision of medical adhesive tapes.

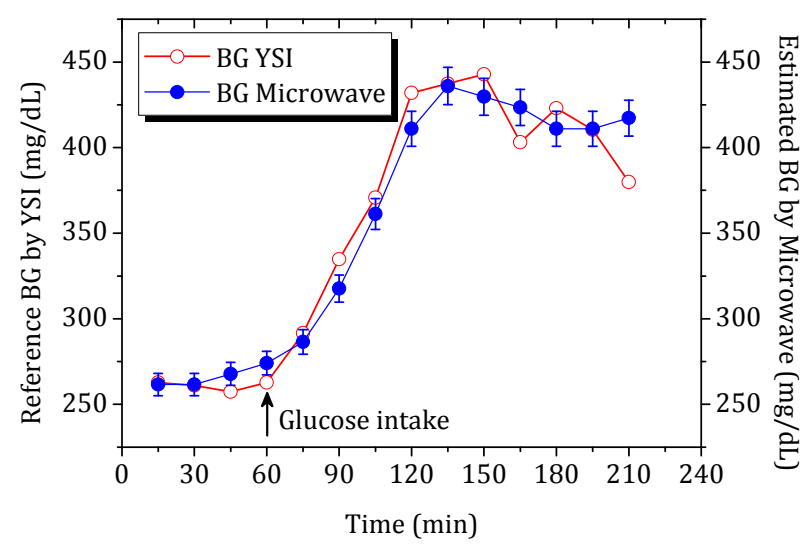

Fig. 3. Oral glucose tolerance test results of subject 18 (type 2), plotted as a function of time and compared with the blood glucose measured at the same time using a laboratory glucose analyzer.

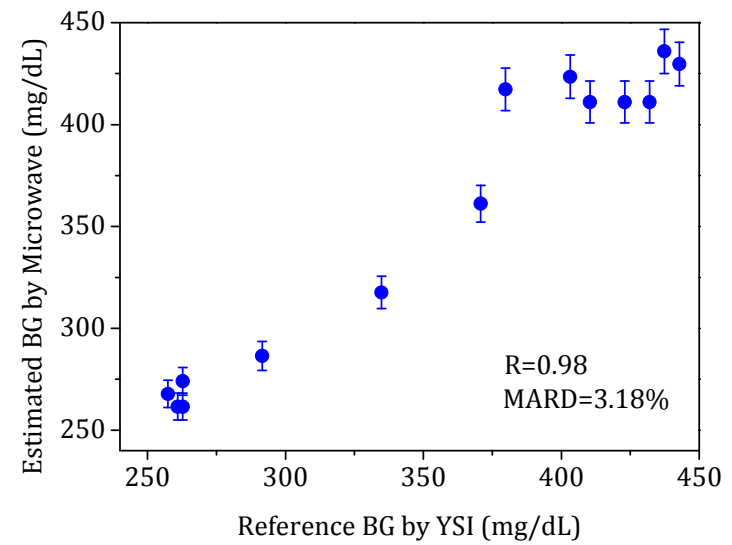

Fig. 4. Scatter chart showing the estimated blood glucose concentration against the reference blood glucose concentration, showing excellent positive correlation between these two data sets.

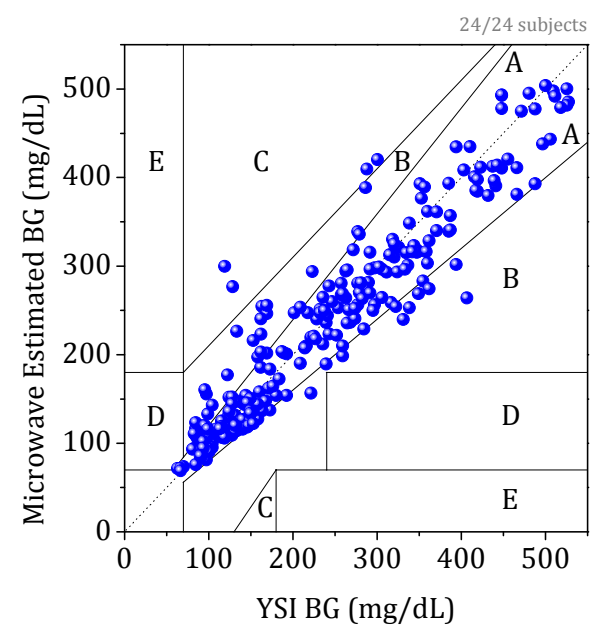

Fig. 5. Clarke Error Grid of data set from 24 subjects (214 data points). 


\section{REFERENCES}

[1] International Diabetes Federation. IDF Diabetes Atlas, 7 ed. Brussels, Belgium: International Diabetes Federation, 2015.

[2] K. Grenier, et al., "Integrated broadband microwave and microfluidic sensor dedicated to bioengineering," IEEE Trans. Microw. Theory Techn., vol. 57, no. 12, pp. 3246-3253, Dec. 2009.

[3] B. R. Jean, E. C. Green, and M. J. McClung, "A microwave frequency sensor for non-invasive blood-glucose measurement," in IEEE Sensors Applications Symp. 2008, Atlanta, GA, 2008, pp. 4-7.

[4] M. Hofmann, G. Fischer, R. Weigel, and D. Kissinger, "Microwave-based noninvasive concentration measurements for biomedical applications," IEEE Trans. Microw. Theory Techn., vol. 61, pp. 2195-2204, May 2013.

[5] T. Yilmaz, R. Foster, and Y. Hao, "Broadband tissue mimicking phantoms and a patch resonator for evaluating noninvasive monitoring of blood glucose levels," IEEE Trans. Antennas Propag., vol. 62, no. 6, pp. 3064-3075, Jun. 2014.

[6] P. H. Siegel, Y. Lee, and V. Pikov, "Millimeter-wave non-invasive monitoring of glucose in anesthetized rats," in 39th Int. Conf. Infrared, Millimeter, and Terahertz waves (IRMMW-THz), Tucson, AZ, Sept. 14-19, 2014, pp. 1-2.

[7] A. Caduff, E. Hirt, Y. Feldman, Z. Ali, and L. Heinemann, "First human experiments with a novel non-invasive, non-optical continuous glucose monitoring system," Biosensors and Bioelectronics, vol. 19, no. 3, pp. 209-217, Nov. 2003

[8] K. Zarkogianni, E. Litsa, K. Mitsis, P. W, C. D. Kaddi, C. Cheng, M. D. Wang, and K. S. Nikita., "A review of emerging technologies for the management of diabetes mellitus," IEEE Trans. Biomedical Engineering, vol. 62, no. 12, pp. 2735-2749, Dec. 2015.

[9] H. Choi, J. Naylon, S. Luzio, J. Beutler, J. Birchall, C. Martin, and A. Porch, "Design and in vitro interference test of microwave noninvasive blood glucose monitoring sensor," IEEE Trans. Microw. Theory Techn., vol. 63, no. 10, pp. 3016-3025, Oct. 2015 .

[10] W. L. Clarke, D. Cox, L. A. Goder-Frederick, W. Carter, S. L. Pohl, "Evaluating clinical accuracy of systems for self-monitoring of blood glucose," Diabetes Care, vol. 10, no. 5, pp. 622-628, 1987. 